\title{
Einkommens- und Vermögensverteilung in Deutschland
}

Die Einkommens- und Vermögensverteilung in Deutschland wird ganz unterschiedlich beurteilt. Je nachdem, welche Indikatoren, welche Stichprobe, welche Datenbasis oder welcher Messzeitpunkt betrachtet werden, kommt es zu einer zunehmenden, stabilen oder abnehmenden Ungleichheit. Die Markteinkommen sind in Deutschland deutlich ungleicher verteilt als die Nettoeinkommen. Das Steuer- und Transfersystem sorgt hier für eine Angleichung - und dies, obwohl die Politik schon seit den 1970er Jahren Steuerreformen durchgeführt hat, die die progressive Wirkung des Steuersystems reduzierten. Eine Untersuchung der Lebenserwerbseinkommen unterschiedlicher Generationen ergibt, dass diese bei jüngeren Einkommensbeziehern ungleicher verteilt sind als bei älteren. Die Bedeutung der Vermögen ist in Zeiten großer Unsicherheit nicht zu unterschätzen, denn Vermögen bietet Sicherheit und Optionen. Letztlich kommt es in Krisenzeiten umso mehr darauf an, das schon seit langem sinkende öffentliche Vermögen stärker in den Fokus zu nehmen.

\section{Verteilungsfragen in Deutschland: Herausforderungen der Messung und der zielgerichteten} Umverteilung

Lars P. Feld, Walter-Eucken-Institut, Albert-Ludwigs-Universität Freiburg und Sachverständigenrat zur Begutachtung der gesamtwirtschaftlichen Entwicklung.

Jan L. Fries, Sachverständigenrat zur Begutachtung der gesamtwirtschaftlichen Entwicklung.

Malte Preuß, Sachverständigenrat zur Begutachtung der gesamtwirtschaftlichen Entwicklung.

Christoph M. Schmidt, RWI - Leibniz-Institut für Wirtschaftsforschung und Ruhr-Universität Bochum.

Steigende Einkommen, sinkende Sorgen - die Zeit vor Corona

Judith Niehues, Institut der deutschen Wirtschaft Köln.

Maximilian Stockhausen, Institut der deutschen Wirtschaft Köln.

Die Ungleichheit von Lebenserwerbseinkommen

Timm Bönke, Freie Universität Berlin.

Holger Lüthen, Freie Universität Berlin.

Vermögensverteilung und Wirtschaftskrisen

Miriam Rehm, Institut für Sozioökonomie der Universität Duisburg-Essen.

Grenzen der Umverteilung im föderalen Sechs-Parteien-Staat

Hermann Adam, Freie Universität Berlin. 